\section{Der Weg vom Ekzem zum Asthma}

Es ist bekannt, dass Kleinkinder mit atopischem Ekzem ein erhöhtes

Risiko für ein Asthma im Alter von sechs Jahren aufweisen. Nun

liegen erstmals epidemiologische Daten bis ins Erwachsenenalter vor.

$\mathrm{n}$ die Tasmanian Longitudinal Health

Study (TAHS) wurden im Jahr 1968 insgesamt 8.583 siebenjährige Schulkinder einbezogen. Anhand eines Fragebogens gaben die Eltern Auskunft über allergische Erkrankungen ihres Kindes, gleichzeitig wurde die Lungenfunktion der Kinder überprüft. 1974 konnten die Eltern von 7.383 Kindern (86\% der ursprünglichen Kohorte) nochmals befragt werden, eine weitere Erhebung zu jemals oder neu aufgetretener Erkrankung an Asthma, Ekzem und saisonaler allergischer Rhinitis erfolgte 2004 mit 5.729 der nun 44 Jahre alten Studienteilnehmer $(81,9 \%$ der ehemaligen Kohorte).

Ein Ekzem in der Kindheit war dabei erwartungsgemäß signifikant assoziiert mit Asthma bronchiale in der Kindheit. Die Assoziation bestand auch noch

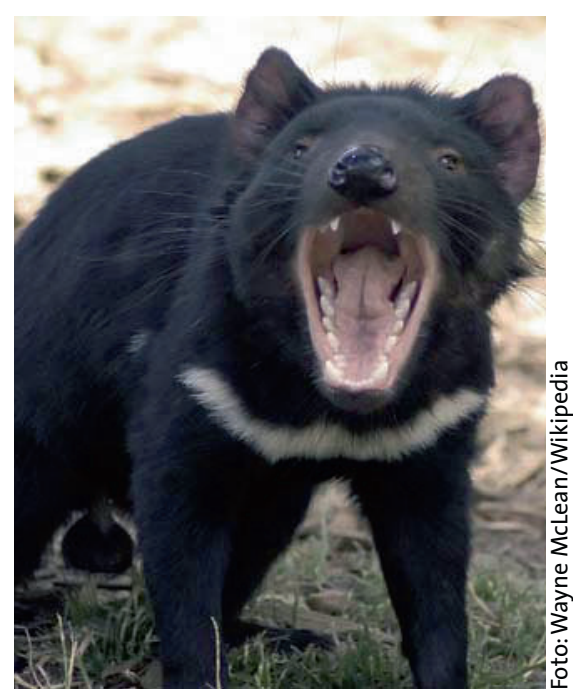

Tasmanien ist nicht nur die Heimat exotischer Beuteltiere, sondern liefert jetzt auch erstmals Daten zum Langzeitasthmarisiko von Kindern mit Ekzem.

\title{
Calcineurininhibitor stärkt Hautbarriere
}

\section{Topische Calcineurininhibitoren hemmen wichtige Funktionen des erworbenen Immunsystems. Jetzt wurde erstmals der Effekt von Pimecrolimus auf das angeborene Immunsystem untersucht.}

Di imecrolimus wird zur Behandlung von atopischer Dermatitis und anderen entzündlichen Hauterkrankungen eingesetzt. Als ein wichtiger Wirkmechanismus wurde bisher die Suppression der T-Zell-Funktionen des erworbenen Immunsystems diskutiert. Die Zielstrukturen der Calcineurininhibitoren, Immunophiline, Calcineurin und NFAT („nucelar factor of activated $\mathrm{T}$ cells"), werden aber auch von Zellen des angeborenen Immunsystems wie den epidermalen Keratinozyten exprimiert. Da Keratinozyten durch die Produktion antimikrobieller, immunmodulierender Peptide (AMPs) wie $\beta$-Defensinen oder Cathelicidinen auch entscheidend an der Aufrechterhaltung der Barrierefunktion der Haut be- teiligt sind, wurde der Einfluss von Pimecrolimus auf die „toll-like“-Rezeptoren (TLR) und andere Immunfaktoren von humanen Keratinozyten in In-vitroExperimenten untersucht.

Es zeigte sich, dass Pimecrolimus in nanomolarer Konzentration die Expression von Cathelicidin, $\beta$-Defensin-2 und $\beta$-Defensin-3 sowie CD14 als Antwort auf eine Aktivierung von TLR2 und TLR6 durch Zugabe entsprechender TLR-Liganden deutlich steigert. Eine weitere Steigerung wurde durch die $\mathrm{Zu}$ gabe von 1,25-Vitamin-D3 erzielt. Pimecrolimus steigerte auch die Fähigkeit von Keratinozyten, das Wachstum von Staphylococcus aureus zu hemmen und reduzierte die TLR2/6-induzierte Expressi- in der Präadoleszenz („hazard ratio“ [HR] 1,70), in der Adoleszenz (HR 2,14) und im Erwachsenenalter (HR 1,63). Allerdings verschwand die signifikante Assoziation zwischen einem Ekzem in der Kindheit und persistierendem Asthma von der Kindheit bis ins mittlere Erwachsenenalter (relatives Risiko 1,54) bei Adjustierung auf das Vorliegen einer allergischen Rhinitis. Insgesamt sind die jetzt erhobenen Befunde ein deutlicher Hinweis darauf, dass sich der „atopische Marsch“ von der Haut in die Schleimhäute bis ins Erwachsenenalter fortsetzen kann.

Fazit: Ein Ekzem in der Kindheit erhöht die Wahrscheinlichkeit für ein Asthma in der Kindheit, aber auch für ein neu auftretendes Asthma im späteren Leben sowie für ein persistierendes Asthma bis ins mittlere Lebensalter.

\section{$b k$}

Burgess JA et al. Childhood eczema and asthma incidence and persistence: a cohort study from childhood to middle age. J Allergy Clin Immunol 2008; 122: 280-5

on von IL-10 und IL-1 $\beta$. Außerdem wurde die nukleäre Translokation von NFAT und NF- $\kappa B$ in den Keratinozyten gehemmt.

Die hier nachgewiesene Aktivierung des angeborenen Immunsystems und die bereits bekannte gleichzeitige Suppression des erworbenen Immunsystems durch Pimecrolimus sind kein Widerspruch, sondern bestätigt aktuelle Befunde, wonach bestimmte regulatorische Mechanismen die Immunsysteme zu teilweise gegensätzlichen Aktionen anregen.

Fazit: In In-vitro-Experimenten steigert Pimecrolimus die Expression von Aktivierungsmarkern des angeborenen Immunsystems. Außerdem wird die Abwehrleistung von Keratinozyten gegen das Wachstum von Staphylococcus aureus verbessert.

Büchau AS et al. Pimecrolimus enhances TLR2/6-induced expression of antimicrobial peptides in keratinocytes. J Invest Dermatol 2008; 128: 2646-54 